\title{
Integrated Active Microfluidics using Flat Panel Display Technology with Two Different Semiconductor Grade Polymers
}

\author{
Boshen Liang $^{12}$, Grim Keulemans ${ }^{1}$, Dominika Wysocka ${ }^{1}$, Alexey Podkovskiy ${ }^{1}$, Lei Zhang ${ }^{1}$, \\ Veronique Rochus ${ }^{1}$, Tim Stakenborg ${ }^{1}$, Paul Heremans ${ }^{12}$, and David Cheyns ${ }^{1}$ \\ ${ }^{1}$ Imec, Leuven, Belgium, boshen.liang@imec.be \\ ${ }^{2}$ KU Leuven, Leuven, Belgium
}

\begin{abstract}
We present an integrated microfluidic system developed based on semiconductor and flat panel display compatible technology to facilitate mass manufacturability of next generation Lab-on-Chip systems. Tailored to different dynamic specifications, two semiconductor grade polymers are selected and used for device fabrication. Compared with PDMS based approach, the selected polymers and optimized excimer laser-aided transferring technique serve as the key factors to enable multi-step and high controllable diagnostic point-of-care platforms with integrated electrical and optical detection schemes.
\end{abstract}

\section{Introduction}

Lab-on-Chip (LOC) has attracted great interest from both the research community and the commercial sector over the past 30 years [1]. As shown in Fig.1, a typical integrated active LOC device consists of fluidic parts represented by microvalves and micropumps to precisely drive and control the routing, timing and mixing of fluids, and of non-fluidic parts represented by electrical and optical components for device control and extra functionalities. The relatively large displacements needed for fluidic components have driven technology developments from bulk and surface micromachining methods to the soft lithography paradigm, where Polydimethylsiloxane (PDMS) like materials enabled fast prototyping and have allowed to reduce the device size two orders of magnitude compared to silicon-based devices [2]. However, the integration of soft-lithography steps such as replica molding in industrial semiconductor fabs or flat panel display (FPD) environment is undesirable for mid and largescale production. Furthermore, it has been a challenge to fabricate electrical and optical components ranging from basic metal contacts to sensors, processors, and actuators directly on top of PDMS-like polymers due to their weak chemical resistance to commonly used solvents and acids in semiconductor and FPD industries [3]. To address these issues, two different semiconductor grade polymers are selected to build up microfluidic devices depending on the different dynamic ranges. Both piezoelectric and pneumatic actuation prototype devices are fabricated and characterized to show the supreme compatibility of the proposed materials and processing methods to semiconductor and flat panel display environment.

\section{Material Choice}

In this study, glass is used as the substrate for its extremely high chemical resistance, high optical transparency and the potential to extend for mass production in flat panel display fabs. SA3990A photoresist and HF-etched quartz are used for $30 \mu \mathrm{m}$ deep microfluidic channels with rectangular or curved cross-sections. For polymers, 2 major factors, the mechanical dynamic range and chemical resistance, are evaluated to make sure the desired functions can be fulfilled, and the processing can be achieved with standard cleanroom methods. Here, the dynamic range refers to the required mechanical deflection to external stimulus. For microvalves where $100 \%$ closure is needed, the polymer should be soft enough to pinch down $30 \mu \mathrm{m}$ fluid channels at reasonable applied forces. On the other hand, for micropumps with nozzle/diffuser as flow rectifying elements, typically a $1 \%$ - 5\% closure ratio of $30 \mu \mathrm{m}$ channel is enough to have effective net flow [4]. The chemical resistance of polymers is checked for common solvents and acids used for direct metal patterning in the cleanroom environment, with representative examples as Ethanol, Butyl acetate, Tetramethylammonium hydroxide, and phosphoric acid. Then, 3 more factors, biocompatibility, optical transparency and gas permeability, are checked for completeness. Based on the material selection criteria, for micropumps Polyimide (PI) is used as the

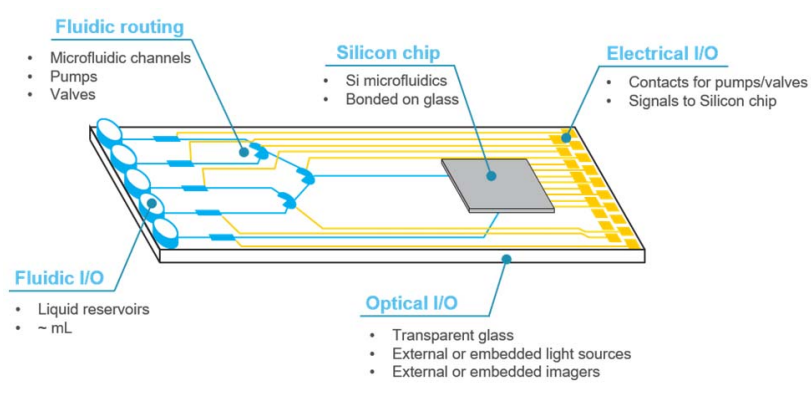

Fig. 1: Schematics of the integrated Lab-on-Chip system consisting of fluid and non-fluid parts. 
Table 1: Material properties of two polymers used in this work compared to that of PDMS

\begin{tabular}{|c|c|c|c|}
\hline Characteristics & PDMS & PI & PIP \\
\hline Young's Modulus & $\sim 1 \mathrm{MPa}$ & $>1 \mathrm{GPa}$ & $\sim 100 \mathrm{MPa}$ \\
\hline Solv. resistance & Weak & Strong & Moderate \\
\hline Acid resistance & Weak & Good & Good \\
\hline Biocompatibility & Good & Good & Good \\
\hline Opt. transparency & Good & Moderate & Moderate \\
\hline Gas permeability & Huge & Small & Small \\
\hline
\end{tabular}

vibrating membrane, and for microvalves Polyisoprene (PIP) is used for a full closure of the fluid channel. Qualitative properties of PI and PIP polymers are measured and listed in Table 1 as compared to reported properties of PDMS [5].

\section{Fabrication}

The process flows of micropumps and microvalves share a great similarity, with the major differences lying in different fluid channel cross-section shapes and different actuation methods. As shown in Fig.2, firstly the PI/PIP resin was spin-coated on a 6-inch donor glass wafer, and then transferred by excimer laser release (SUSS XBC300 Gen2 Debonder) to a 6" glass or quartz wafer with either SA3990 or HFetched etched $30 \mu \mathrm{m}$ deep fluid channels. The laser dose was optimized to be $160 \mathrm{~mJ} / \mathrm{cm}^{2}$ from a balance

\section{PI/PIP spinning}

6"' Donor Glass

2. Wafer level bonding and laser delamination

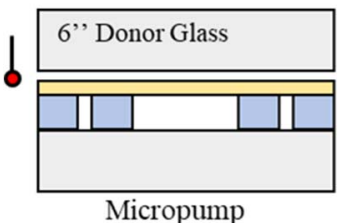

3. Top part preparation

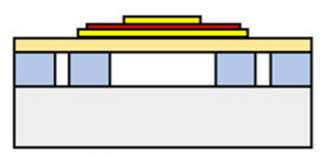

Micropump
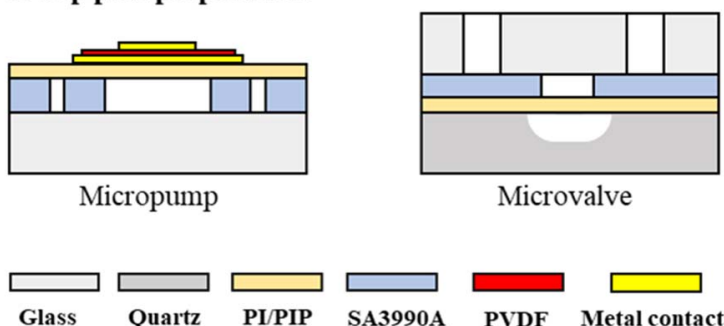

Microvalve

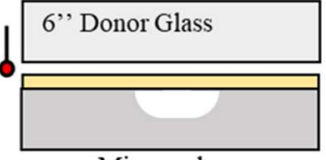

Microvalve

Fig. 2: Fabrication process of the microfluidic system. The micropump and microvalve process flows share similarity concerning the preparation and transfer of polymer, while they are different for fluid channel cross-section shapes and actuation methods.

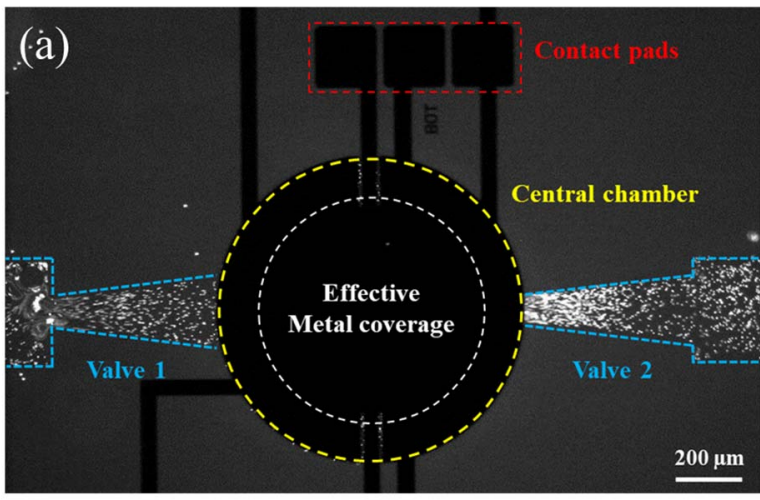

(b)

(c)

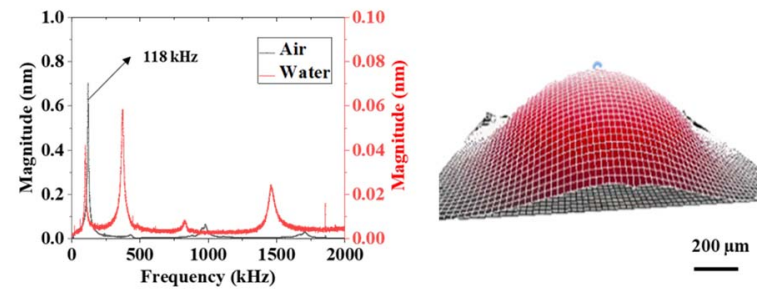

Fig. 3: Characterization of fabricated piezoelectric micropump. (a) MicroPIV test image. The florescent microspheres aggregate to the right side of the micropump, which illustrates a net flow from left to right side. (b) Frequency response of the fabricated micropump with or without fluid channel filled with water. (c) 3D reconstructed shape of the vibrating membrane when the fluid channel is filled with water.

of good debonding and minimum residue. Then, for piezoelectric micropumps, $60 \mathrm{~nm}$ aluminum was deposited and patterned on top of PI as bottom contact. P(VDF-TrFE) powder was dissolved in Methyl Ethyl Ketone and the solution was spincoated on top of the wafer to form a $0.5 \mu \mathrm{m}$ piezoelectric layer, which was later cured in vacuum and $\mathrm{N}_{2}$ atmosphere. Another $60 \mathrm{~nm}$ aluminum layer was deposited and patterned as top metal contact. For pneumatic microvalves, another glass is preperforated and laminated with patterned $50 \mu \mathrm{m}$ thick SA3990 dry film photoresist as air channel. The fluid channel and air channel are bonded together to form the microvalve device.

\section{Results and Discussion}

A. Piezoelectric micropump with Polyimide polymer A microscope image of the piezoelectric micropump is shown in Fig.3(a). Each micropump unit occupies less than $1.5 \mathrm{~mm} \times 1 \mathrm{~mm}$ area on glass wafer. Laser Doppler vibrometer (LDV, Polytec MSA500) was used for membrane vibration characterization. Firstly, a high frequency unit was used to measure the 
(a)

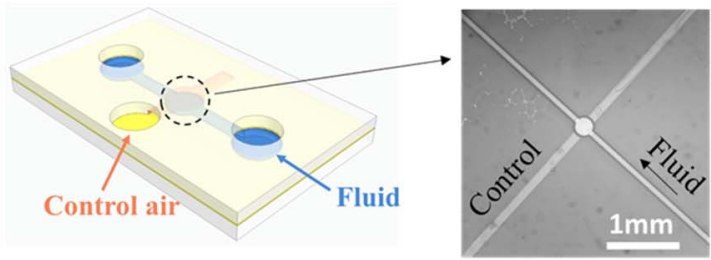

(b)

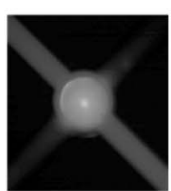

$5 \mathrm{kPa}$

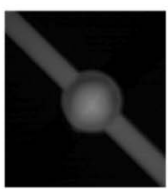

$20 \mathrm{kPa}$

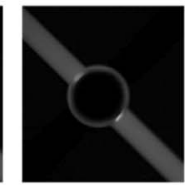

$50 \mathrm{kPa}$

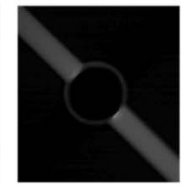

$85 \mathrm{kPa}$

(c)

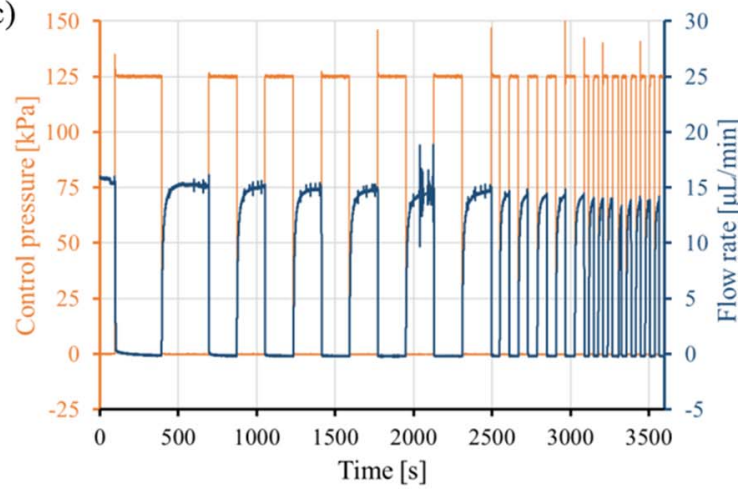

Fig. 4: 3D schematic of the assembled microvalve chip (Left) and an example of microscope images of a $250 \mu \mathrm{m}$ diameter circular normally open pneumatic valve (Right). (b) Fluorescence images displaying the closure of the microvalve when increasing the control pressure from 5 to $85 \mathrm{kPa}$ (c) 20 cycles of a $250 \mu \mathrm{m}$ circular microvalve at different intervals (30 sec to $5 \mathrm{~min}$ ), with fluidic pressure at inlet was $50 \mathrm{kPa}$.

vibration of the PI membrane with and without the channel filled with water. In the air, the resonance peak at $118 \mathrm{kHz}$ corresponds to the $1 \mathrm{st}$ mode vibration as shown in Fig. 3(b). To capture the $1^{\text {st }}$ mode with filled water, the LDV was switched to low frequency measurements. As shown in Fig.3(c), the $1^{\text {st }}$ mode vibration of the membrane changed to 4.4 $\mathrm{kHz}$ and was reconstructed in 3D form. Micron resolution particle image velocimetry (Micro-PIV) was used for micropump flowrate characterization. Carboxylate-modified microspheres (FluoSpheres ${ }^{\mathrm{TM}}$, $2.0 \mu \mathrm{m}$ diameter, $515 \mathrm{~nm}$ fluorescence peak) was diluted in water and then injected in the fluid channel, and an inverted microscope setup with a high-speed camera was used to capture the movement of the microspheres. As shown in Fig. 3(c), after turning on the driving voltage, the fluorescent microspheres start aggregating towards the right side of the micropump, which illustrates a net flow from left to right side.

\section{B. Pneumatic microvalve with Photoresist polymer}

A 3D schematic of the assembled microvalve and a microscope image of a $250 \mu \mathrm{m}$ diameter circular valve is shown in Fig.4(a). An optical inspection of the fluid flow was performed with a high-speed camera. Quantitative flow data was captured with a flow unit at the valve outlet. The flow is blocked when applying a control pressure of $85 \mathrm{kPa}$ for a fluidic pressure of $15 \mathrm{kPa}$ as shown by the fluorescence microscope images in Fig.4(b). Dynamically, Stable and repeatable opening/closing operations of the valve are demonstrated over multiple cycles for tested flow pressure from $5-50 \mathrm{kPa}$ and control pressures in the range of 50-125 $\mathrm{kPa}$. A typical multicycle operation of the microvalve is shown in Fig.4(c), indicating good reliability of the device.

\section{Conclusion}

An integrated Lab-on-chip system with great compatibility with industrial semiconductor and flat panel display technology is presented. Two different semiconductor grade polymers are used for fluidic components that cover different dynamic ranges. Verified for both piezoelectric and pneumatic schemes with standard cleanroom facilities, our proposed concepts and techniques are not restricted in labs but have the potential for mass production in fabs in the mature phase.

\section{References}

[1] P. Abgrall and A. M. Gué, "Lab-on-chip technologies: Making a microfluidic network and coupling it into a complete microsystem - A review," Journal of Micromechanics and Microengineering, vol. 17. p. R15, May-2007.

[2] M. A. Unger, H. P. Chou, T. Thorsen, A. Scherer, and S. R. Quake, "Monolithic microfabricated valves and pumps by multilayer soft lithography," Science (80-. )., vol. 288, no. 5463, pp. 113-116, Apr. 2000.

[3] W. H. Grover, A. M. Skelley, C. N. Liu, E. T. Lagally, and R. A. Mathies, "Monolithic membrane valves and diaphragm pumps for practical largescale integration into glass microfluidic devices," Sensors Actuators B Chem., vol. 89, no. 3, pp. 315323, 2003.

[4] A. Ullmann and I. Fono, "The Piezoelectric ValveLess Pump- Improved dynamic model," vol. 11, no. 6, pp. 655-664, 2002.

[5] A. Mata, A. J. Fleischman, and S. Roy, "Characterization of Polydimethylsiloxane (PDMS) Properties for Biomedical Micro/Nanosystems," Biomed. Microdevices, vol. 7, no. 4, pp. 281-293, Dec. 2005. 